\title{
Global scattering functions: a tool for grazing incidence small angle X-ray scattering (GISAXS) data analysis of low correlated lateral structures
}

\author{
S. Lenz ${ }^{1}$, M. Bonini ${ }^{2}$, S.K. Nett ${ }^{1,3}$, M.C. Lechmann ${ }^{1}$, S.G.J. Emmerling ${ }^{1}$, R.S. Kappes ${ }^{1}$, M. Memesa $^{1}$, A. Timmann $^{4}$, \\ S.V. Roth ${ }^{4}$, and J.S. Gutmann 1,3, a \\ 1 Max-Planck Institute for Polymer Research, Ackermannweg 10, 55128 Mainz, Germany \\ 2 Department of Chemistry and CSGI, University of Florence, Via della Lastruccia 3, 50019 Sesto Fiorentino, Florence, Italy \\ 3 Institute for Physical Chemistry, Johannes Gutenberg University, Jakob-Welder-Weg 10, 55099 Mainz, Germany \\ 4 HASYLAB at DESY, Notkestr. 85, 22603 Hamburg, Germany
}

Received: 16 March 2009 / Received in final form: 14 September 2009 / Accepted: 1st April 2010 Published online: 17 June 2010 - (C) EDP Sciences

\begin{abstract}
In this article we discuss the applicability of global scattering functions for structure analysis of Grazing Incidence Small Angle X-ray Scattering (GISAXS) data. Contrary to rigorous analysis of the full 2-D detector image, which can be performed with complex simulation models, the global scattering functions described here will be used to model transverse detector scans in the $q_{\|}$reciprocal scattering planes. In contrast to a full GISAXS analysis, this procedure cannot explain structural features perpendicular to the sample plane. The discussed method is useful for the analysis of weakly correlated films. These films are e.g. found in polymer inorganic composite materials based on commercially available nanoparticles. In hybrid material systems polydisperse structures, including particle aggregates without precisely defined shape are formed. The pictured approach, which models scattering in terms of structural levels, has been previously applied with success in conventional transmission SAXS geometry. It is based on conventional exponential and power laws. Hence, data analysis becomes less complex compared to simulation approaches. Here we examine if this unified fitting model can be used to model diffuse, non specular scattering resulting from GISAXS. In this context the applicability and limit of its application to diffuse scattering in the GISAXS geometry is discussed. Furthermore diffuse $q_{\|}$scattering from different ideal particle types is simulated and compared with fitted results. To verify our approach, fit results from experimental GISAXS curves obtained for real samples are compared with results from Scanning Probe Microscopy and Scanning Electron Microscopy studies. The samples investigated range from evaporated $\mathrm{Au}$ films to hybrid $\mathrm{TiO}_{2} /$ polymer films and demonstrate the usefulness in the structural analysis of complex films.
\end{abstract}

\section{Introduction}

Grazing incidence small angle X-ray scattering (GISAXS) can probe structural film properties perpendicular and parallel to the sample plane. Information on film structures perpendicular to the sample plane is usually obtained by the analysis of the specular reflected beam in $q_{\perp}$ direction. In addition lateral film structures can be studied by either analyzing scattering parallel to the sample's surface at the specular beam position or by analyzing additional off specular scattering.

Our aim is to probe lateral length scales ranging from a few nanometres up to hundreds of nanometres $[1,2]$ of colloidal systems with a defined mean particle dimension and typical polydispersities with standard deviations $\sigma=0-0.6$, which is often sufficient to understand material film properties [3]. Polydisperse particles can be

\footnotetext{
a e-mail: gutmann@mpip-mainz.mpg.de
}

located as islands at the film/air interface or buried within a thin film matrix. Especially for buried particle systems high penetration depths into the film matrix are recommended. Thus, incident angles $\alpha_{i}$, which are sufficiently higher than the critical angle $\alpha_{c}$ of the film matrix, should be chosen. We demonstrate under which conditions our approach yields results for mean particle dimensions with deviations in the range of the particles polydispersity at GISAXS taken at $\alpha_{i}>\alpha_{c}$.

At these experimental conditions, it is possible to benefit from a separation of the reflected intensity into two peaks in $q_{\perp}$ direction. One is the specular reflected beam at an angle of the exit beam $\alpha_{f}$ equal to $\alpha_{i}$. The second is the material dependent Yoneda peak $[4,5]$ at $\alpha_{f} \approx \alpha_{c}[6,7]$. Diffuse scattering at this peak position can be explained within the distorted wave born approximation (DWBA) [8]. For practical reasons a separation of the two peak positions is useful, because scattering at the specular beam position, can be separated from diffuse 
scattering occurring at the Yoneda peak. Hence, deconvolution of the primary reflected beam profile, scattering at the specular peak position and diffuse scattering at the Yoneda peak can be avoided.

Transverse detector scans along the reciprocal $q_{\|}\left(q_{x}, q_{y}\right)$ scattering plane at the Yoneda peak position can be performed to obtain lateral film information. Occurring peaks on the transverse $q_{\|}$scans are often analyzed with a generalized Bragg interference function $\xi_{\|}=\frac{2 \pi}{q_{\|}}$, which is widely used for Born approximated scattering. However, for a rigorous data analysis of GISAXS one should use the DWBA. Theories for the most important film systems containing rough surfaces [8-10], buried particles [11] and supported islands [12] were previously derived. To compute and simulate GISAXS data, Lazzari developed the simulation and fitting software IsGISAXS ${ }^{1}[13,14]$. In this software the mentioned theories and approximations are included.

Nevertheless, simulation of scattering data will only be meaningful if additional information on the film system is available, including an exact model of the particle shape and lattice. In cases of film systems composed of typical polydisperse colloidal particles, which are not arranged on a grid, the use of well defined form and structure factors will become questionable.

Comparable weakly correlated colloidal powder systems and polymer solutions were analysed in the past with unified fit approaches in transmission geometry [15-18]. This model describes scattering from a specimen in terms of multiple structural levels. It is based on a combination of exponential laws, power laws and Bragg based interference functions and describes fractal scattering objects by their fractal dimensions, radius of gyrations and mean centre to centre distances. Compared to full DWBA simulation approaches, a unified approach would make GISAXS data analysis of the latter film systems easier. However, before the application of such BA based models, it is of high importance to discuss the applicability and limits towards DWBA described $q_{\|}$scattering in detail.

Our considerations combine theoretical discussions with simulation and experimental studies. Comparisons of simulations computed for BA based transmission SAXS and DWBA based GISAXS demonstrate the principal applicability of the unified formalism to GISAXS. Based on these comparisons, an optimum experimental range for the incident angle is proposed for particle island systems at the film/air interface and buried particle systems. While in this range refraction effects cannot be neglected, it is nevertheless possible to obtain fit results deviating less than $10 \%$ from their theoretical values for monodisperse particle samples. For polydisperse film systems mean deviations are not exceeding $\sigma$ or usual experimental error intervals.

Experimental GISAXS studies were performed on polydisperse $\mathrm{Au}$ particle islands prepared by chemical vapour deposition, as a sample system for polydisperse

\footnotetext{
1 www.insp.jussieu.fr/axe2/0xydes/IsGISAXS/ isgisaxs.htm
}

particles located at a free interface. The practical usefulness of the unified fit approach is further demonstrated for buried particles. The study of a model $\mathrm{TiO}_{2} /$ polymethylmethacrylate (PMMA) hybrid material film demonstrates the applicability of the unified fit approach to buried particle systems. The usefulness of unified fits to unravel new physical phenomena is demonstrated in an investigation of percolating networks in hybrid barrier layers used in solar cell applications. Here the analysis of GISAXS measurements using the unified fit approach is able to prove the existence of fractal 3D networks proposed from conductive SPM (scanning probe microscopy) experiments [19]. It therefore establishes a structural model which explains the physics of charge carrier transport in these systems.

\section{Experimental methods}

GISAXS with the help of $\mu$-sized X-ray beams was performed on three exemplary film systems. First the adaptability of the unified fit approach towards $q_{\|}$scattering on rough $\mathrm{Au}$ films, with low lateral correlations shall be studied. To this end we prepared one Au film of a thickness of $20 \mathrm{~nm}$ deposited on a base cleaned Si-substrate by thermal evaporation methods (BALTEC MED 020, BALTEC, Balzers, Lichtenstein), which was carried out under a chamber base pressure of $1.7 \times 10^{-5}$ mbar with an evaporation rate of $<1 \AA / \mathrm{s}$ and room temperature substrates. GISAXS on a film of polydisperse $\mathrm{TiO}_{2}$ particles buried in a polymer matrix was performed, in order to demonstrate the usefulness of the unified model for buried particle systems. $\mathrm{TiO}_{2}$ particles $(0.01 \mathrm{ml}$, Solaronix $\mathrm{T})$ were dispersed in a PMMA $(0.1 \mathrm{~g})$ solution in THF $(5 \mathrm{ml})$, which resulted in a film with $\sim 1 \%$ wt $\mathrm{TiO}_{2}$. The film was spin coated on Si substrates for $60 \mathrm{~s}$ using a Süss Micro Tec Delta 80 spin coater under ambient conditions (rotation speed of $1000 \mathrm{rpm}$; acceleration speed of $1000 \mathrm{rpm} / \mathrm{s}$ ). The thickness of the studied film was $180 \mathrm{~nm} \pm 10 \mathrm{~nm}$, as measured with a Tencor P-10 surface profiler. In addition, one percolating $\mathrm{TiO}_{2}$ hybrid material film was prepared by sol-gel-chemistry using a templating (PEO)MA-PDMS-MA(PEO) tri-blockco-polymer. The film was plasma treated and ceramized at $600{ }^{\circ} \mathrm{C}$ as described previously [19]. The thickness of the studied film was $20 \pm 1 \mathrm{~nm}$, as measured by X-ray reflectivity with a Seifert $\theta-\theta$ XRD 3003 X-ray reflectometer.

GISAXS experiments were carried out at the beamline BW4/HASYLAB using the microfocus option [20-22], with a sample-to-detector distance of $1.9 \mathrm{~m}$, a wavelength $\lambda=0.138 \mathrm{~nm}$, and a beam size of $32 \times 17 \mu \mathrm{m}^{2}$ (horizontal $\times$ vertical). A compromise between a sufficient separation of specular reflected peaks and Yoneda peaks, a minimized theoretical deviation between DWBA and BA theory (see Sect. 3) and available experimental conditions was made. Consequently an incident angle of $\alpha_{i}$ equal to $0.7^{\circ}$ was chosen for GISAXS studies on the Au film. GISAXS on the $\mathrm{TiO}_{2} / \mathrm{PMMA}$ film GISAXS scattering patterns were measured at $\alpha_{i}=0.55^{\circ}$, while $\mathrm{TiO}_{2} /(\mathrm{PEO}) \mathrm{MA}-$ PDMS-MA(PEO) films were measured at $\alpha_{i}=0.7^{\circ}$. 
Transverse $q_{\|}$scans were performed at the Yoneda's maximum at $\alpha_{f} \approx \alpha_{c}(\mathrm{Au})=0.50$ for the measured $\mathrm{Au}$ film, $\alpha_{f} \approx \alpha_{c}(\mathrm{PMMA})=0.14$ for the $\mathrm{TiO}_{2} / \mathrm{PMMA}$ film and $\alpha_{f} \approx \alpha_{c}((\mathrm{PEO}) \mathrm{MA}-\mathrm{PDMS}-\mathrm{MA}(\mathrm{PEO}))=0.14$ for the $\mathrm{TiO}_{2} /(\mathrm{PEO}) \mathrm{MA}-\mathrm{PDMS}-\mathrm{MA}(\mathrm{PEO})$.

In order to compare GISAXS data and the obtained fit parameters with comparative methods, SPM and SEM studies were performed on $\mathrm{Au}$, pure $\mathrm{TiO}_{2}$ particle films and $\mathrm{TiO}_{2} /(\mathrm{PEO}) \mathrm{MA}-\mathrm{PDMS}-\mathrm{MA}(\mathrm{PEO})$, respectively.

$\mathrm{SPM}$ images of the $\mathrm{Au}$ films were taken in tapping mode at constant amplitude (environmental SPM, Veeco Instruments, Santa Barbara, CA). For these studies silicon cantilevers (Olympus, OMCL-AC240TS, Japan) having a nominal spring constant of $2 \mathrm{~N} / \mathrm{m}$ and a resonance frequency of around $70 \mathrm{kHz}$ were used. For operation of the SPM we used a NanoScope IIIa controller (Veeco Instruments, Santa Barbara, CA, USA) controlled by Nanoscope $5.30 \mathrm{r} 2$ software. The offset and tilt background of all images were removed by subtraction of a 1st and 2nd polynomial background due to scanner movement. The size of immersed $\mathrm{TiO}_{2}$ particles (without polymer matrix) and of the $\mathrm{TiO}_{2} /(\mathrm{PEO}) \mathrm{MA}-\mathrm{PDMS}-\mathrm{MA}(\mathrm{PEO})$ was assigned using a field emission SEM (LEO 1530 "Gemini"). The acceleration voltage was $1 \mathrm{kV}$.

\section{Results and discussion}

\subsection{Theoretical discussion}

\subsubsection{GISAXS geometry}

In a typical GISAXS experiment the primary beam is directed in a certain incident angle $\alpha_{i}$ onto the sample's surface. The incident beam is reflected and scattered at the samples surface, with an exit angle $\alpha_{f}$ perpendicular to the surface and an angle $2 \theta$ parallel to the surface (Fig. 1). The scattering wave vector $q$ is composed of its single components $q_{x}, q_{y}$ and $q_{z}$, related to the experimental angles by

$$
q=\left(\begin{array}{c}
q_{x} \\
q_{y} \\
q_{z}
\end{array}\right)=\frac{2 \pi}{\lambda}\left(\begin{array}{c}
\cos \left(\alpha_{f}\right) \cos (2 \theta)-\cos \left(\alpha_{i}\right) \\
\cos \left(\alpha_{f}\right) \sin (2 \theta) \\
\sin \left(\alpha_{f}\right)+\sin \left(\alpha_{i}\right)
\end{array}\right)
$$

When absorption in the samples medium cannot be neglected, $q_{z}$ becomes complex and is related to the imaginary part, $\beta$ of the refractive index $n=1-\delta+i \beta$ and the materials critical angle $\alpha_{c}=\arcsin (\sqrt{2 \delta}) \approx \sqrt{2 \delta}$ by $[23,24]$

$$
\begin{aligned}
\tilde{q}_{z}=\frac{2 \pi}{\lambda}\left(\sqrt{\sin ^{2} \alpha_{i}-\sin ^{2} \alpha_{c}+2 i \beta}\right. & \\
& \left.+\sqrt{\sin ^{2} \alpha_{f}-\sin ^{2} \alpha_{c}+2 i \beta}\right) .
\end{aligned}
$$

Throughout the rest of this work we use the $q_{\perp}=$ $\sqrt{q_{x}^{2}+q_{z}^{2}}$ and $q_{\|}=\sqrt{q_{x}^{2}+q_{y}^{2}}$ reciprocal planes to describe scattering, reflection and refraction perpendicular and parallel to the specimen's surface.

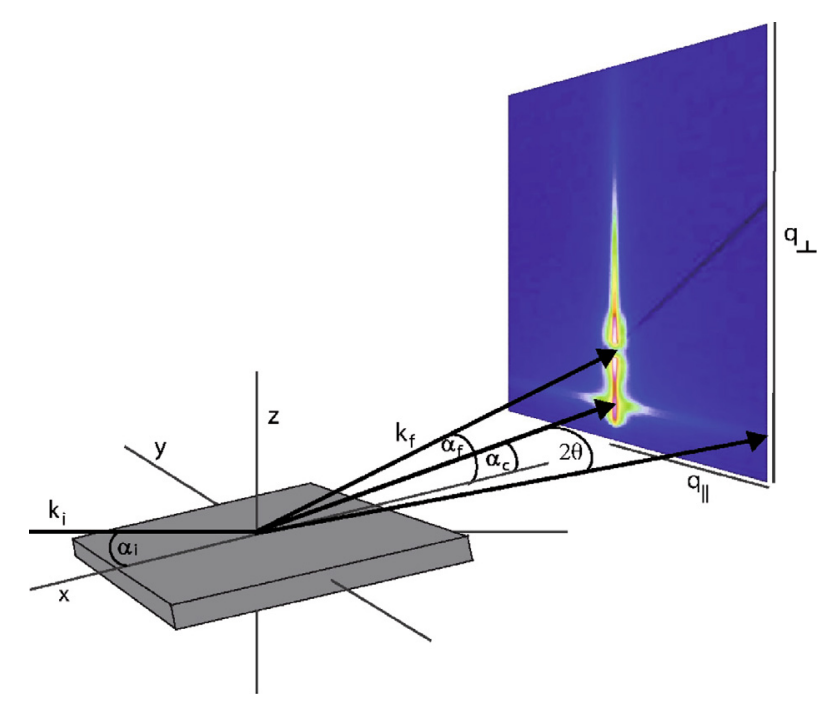

Fig. 1. (Color online) GISAXS geometry.

\subsubsection{Approximation of diffuse scattering by BA} and intrinsic limits

In order to reduce GISAXS analysis to lateral density fluctuations, detector scans in $q_{\|}$are commonly performed at the Yoneda's maximum at $\alpha_{f}$ or $\alpha_{i}$ equal to $\alpha_{c}$ of one film component [5]. When analyzing transverse detector scans with conventional BA, the averaged intensity in the DWBA, which describes the four major scattering effects (curly brackets in Eq. (3)) has to be considered [11].

$$
\begin{aligned}
& \left\langle\left|\Psi^{2}\right|\right\rangle=\left\{\left|\Psi^{(0)}+\left\langle\Psi_{S}\right\rangle+\left\langle\Psi_{d}\right\rangle\right|^{2}\right\}+\left\{\left\langle\left|\Psi_{S}\right|^{2}\right\rangle-\left|\left\langle\Psi_{S}\right\rangle\right|^{2}\right\} \\
& +\left\{\left\langle\left|\Psi_{d}\right|^{2}\right\rangle-\left|\left\langle\Psi_{d}\right\rangle\right|^{2}\right\}+\left\{2 \operatorname{Re}\left(\left\langle\Psi_{S} \Psi_{d}^{*}\right\rangle-\left\langle\Psi_{S}\right\rangle\left\langle\Psi_{d}^{*}\right\rangle\right)\right\}
\end{aligned}
$$

$\Psi^{(0)}$ denotes the specular reflected amplitude from a smooth film surface without interior density fluctuations. The subscript $S$ denotes the surface, while $d$ denotes density fluctuations of the film's interior. $\left|\Psi^{(0)}+\left\langle\Psi_{s}\right\rangle+\left\langle\Psi_{d}\right\rangle\right|^{2}$ describes the scattered intensity at the specular beam position, $\left\langle\left|\Psi_{s}\right|^{2}\right\rangle-\left|\left\langle\Psi_{s}\right\rangle\right|^{2}$ describes diffuse scattering from the surface roughness, $\left\langle\left|\Psi_{d}\right|^{2}\right\rangle-\left|\left\langle\Psi_{d}\right\rangle\right|^{2}$ can be correlated to diffuse scattering from density fluctuations within the medium. The last term $2 \operatorname{Re}\left(\left\langle\Psi_{S} \Psi_{d}^{*}\right\rangle-\left\langle\Psi_{S}\right\rangle\left\langle\Psi_{d}^{*}\right\rangle\right)$ includes possible correlations between scattered waves from surface roughness and density fluctuations and can in many cases be approximated to 0 . With proper selection of $\alpha_{i}$ and $\alpha_{f}$, minimization of refraction effects and separation of scattering at the specular beam position and nonspecular, diffuse scattering at the Yoneda peak can be achieved. Thus it becomes possible to treat term one independently from term two and three $[11,25]$. However, the non-specular scattered intensity is still a sum of scattered intensities resulting from surface roughness and interior density fluctuations.

Sinha et al. [8] derived the differential cross-section of diffuse scattering as

$$
\frac{d \sigma}{d \Omega}=\frac{A k_{c}^{2}}{(4 \pi)^{2}}\left|T^{i} T^{f}\right|^{2} \Gamma(q)
$$


where $A$ is the irradiated sample surface area, $k_{c}^{2}$ is the critical impulse and $T^{i}, T^{f}$ the Fresnel transmission coefficient of the incoming and outcoming wave, respectively, given by

$$
T=\frac{2 k_{\perp}}{k_{\perp}+\tilde{k}_{\perp}}
$$

Here $k_{\perp}$ and $\tilde{k}_{\perp}$ are the wave vectors perpendicular to the sample surface in vacuum and in the film medium, respectively. $\Gamma(q)$ can be related to density autocorrelation functions, which describe the form of idealized scattering objects. To deconvolute $\Gamma(q)$ Rauscher et al. [11] used an infinitely thin and perfectly flat $\delta$-layer with interior density fluctuations of a lateral distance $R_{\|}$and obtained

$$
\Gamma(q)=\int d^{2} R_{\|} \exp \left(-i q_{\|} \cdot R_{\|}\right) C_{w}\left(R_{\|}\right) t^{2}=\hat{C}_{w}\left(q_{\|}\right) t^{2}
$$

where $\hat{C}_{w}\left(q_{\|}\right)$is the Fourier transform of the density autocorrelation function $C_{w}\left(R_{\|}\right)$of the lateral surface structures within this $\delta$-layer, and $t$ is the thickness of the $\delta$-layer. In the case of non correlated density fluctuations, $\hat{C}_{w}\left(q_{\|}\right)$can be substituted with an idealized particle form factor. It has to be noted that the lateral approximation in equation (6) is only valid for infinitely thin cylindrically symmetric scattering objects. From $\hat{C}_{w}$ of spherical particle islands [13].

$$
\hat{C}_{w}^{\text {Sphere }}(q, R)=4 \pi R^{3} \frac{\sin (q R)-q R \cos (q R)}{(q R)^{3}} \exp \left(i q_{z} R\right)
$$

one can see that GISAXS in the $q_{\|}$detector plane will have a non constant $q_{\perp}$ scattering proportion. Nevertheless, it is shown later on that a lateral approximation for $\hat{C}_{w}^{\text {Sphere }}(q, R)$ leads to deviations below $10 \%$ for monodisperse particle samples. For polydisperse film systems mean deviations are not exceeding $\sigma$ or usual experimental error intervals, which is sufficient for the analysis of typical polydisperse colloidal particles.

At this point an expression is needed, which includes scattering from rough interfaces coupled with scattering from objects with different scattering length densities. Apart from height-height correlation functions $C_{h}\left(\left|r_{\|}^{\prime}-r_{\|}^{\prime \prime}\right|\right)=\left\langle h\left(r_{\|}^{\prime}\right) h\left(r_{\|}^{\prime \prime}\right)\right\rangle$, which return root mean square (rms) values, lateral roughness correlations at $q_{\|} \neq 0$ can be studied. Characteristic Porod decays, $I \sim q^{-3-h}$, is used to describe the fractality or self affinity of the rough surface or interface. The Hurst parameter $h$ is equal to unity for smooth surfaces or very small for jagged surfaces $[8,26-28]$. These surfaces exhibit a lateral cut-off length $\zeta$. Considering a self-affine rough particle island of an arbitrary shape, which can be supported on a film or buried in a film (e.g. polymer) matrix, it becomes appropriate to associate the cut-off length of this rough structure with its radius of gyration $R_{g}$.

\subsubsection{Unified exponential/power-law fit mode}

At this point an expression is needed, which is capable of describing simultaneously scattering from rough interfaces and density fluctuations. In this context density fluctuations shall be described with different idealized shapes. Beaucage introduced in a set of publications $[16,17,29]$ a general unified fit model, which is able to describe scattering over several orders of magnitude for spherical averaged particles in transmission geometry using BA. This model describes material microstructures in terms of structural levels. Thus it can be applied to model the system's structural features starting from the smallest structural level, such as a nanoparticle towards clusters of particles up to the macro-scale. It was applied successfully in transmission scattering geometry to several particle systems [30-32]. In addition, it was also applied to scattering from soft matter systems $[29,33,34]$. Nevertheless, this global model was originally developed for analysis of scattering results in transmission geometry using BA. Therefore we have to show that it is (within certain limitations) also applicable to the analysis of scattering problems in grazing incidence geometry.

For one structural level the scattered intensity in the unified fit approach is given by

$$
I(q)=G \exp \left(-\frac{q^{2} R_{g}^{2}}{3}\right)+B\left[\frac{\left(\operatorname{erf}\left(q R_{g} / \sqrt{6}\right)\right)^{3}}{q}\right]^{P} .
$$

The first term corresponds to Guinier's law and describes the size of spherical averaged particles. It is related to the radius of a sphere by $R_{g}=\sqrt{\frac{3}{5}} R$. For upstanding particles with cylindrical symmetries, $q$ can be substituted with $q_{\|}$ and term one in equation (8) with $G \exp \left(-\frac{q_{\|}^{2} R_{c}^{2}}{2}\right) . R_{C}$ is defined as the radius of gyration of the cross-sectional area of the particle and can be related to the radius of the crosssectional area by $R_{C}=\frac{1}{\sqrt{2}} R_{\|}$.

The second term in equation (8) corresponds to the structural limited Porod regime, with the Porod prefactor $B$. The cubed error function limits the fractal regime of the structure's surface at low $q$ over three possible orientations with its radius of gyration $R_{g}$. For smooth spherical particles $q^{-4}$ dependence is obtained, while for surface scattering from cylindrically symmetrical structures $q^{-3}$ dependence is obtained [35].

For $q_{\|}$scattering from cylindrically symmetric particles equation (8) can be rewritten with a squared error function allowing a power law cut-off over two orientations

$$
I\left(q_{\|}\right)=G \exp \left(-\frac{q_{\|}^{2} R_{C}^{2}}{2}\right)+B\left[\frac{\left(\operatorname{erf}\left(q_{\|} R_{g} / \sqrt{6}\right)\right)^{2}}{q_{\|}}\right]^{P} .
$$

When more than one structural level is present in a sample, the unified scattering intensity described in 
equation (8) can be extended to [16]:

$$
\begin{array}{r}
I(q) \approx \sum_{i=1}^{n} G_{i} \exp \left(\frac{-q^{2} R_{g i}^{2}}{3}\right)+B_{i} \exp \left(\frac{-q^{2} R_{g(i-1)}^{2}}{3}\right) \\
\times\left[\frac{\left(\operatorname{erf}\left(q R_{g i} / \sqrt{6}\right)\right)^{3}}{q}\right]^{P} \cdot
\end{array}
$$

Equation (10) is a sum of scattered intensities over $n$ structural levels. When there are correlations between two structural levels, as in particle aggregates, a second term, which limits Porod scattering for $n>1$ at $R_{g(i-1)}$ has to be introduced. For $q_{\|}$scattering of cylindrically symmetric structures an analogue expression can be written

$$
\begin{gathered}
I\left(q_{\|}\right) \approx \sum_{i=1}^{n} G_{i} \exp \left(\frac{-q_{\|}^{2} R_{c i}^{2}}{2}\right)+B_{i} \exp \left(\frac{-q^{2} R_{c(i-1)}^{2}}{2}\right) \\
\times\left[\frac{\left(\operatorname{erf}\left(q_{\|} R_{g i} / \sqrt{6}\right)\right)^{2}}{q_{\|}}\right]^{P} \cdot
\end{gathered}
$$

In SAXS analysis the Guinier and Porod prefactors $G$ and $B$, can be related to the polydispersity index (PDI) of spherical averaged particles in SAXS by $P D I=$ $B R_{g}^{4} /(1.62 G)$ [36]. However, simulations presented later show that such PDI approaches are not valid when applied to $q_{\|}$scans in GISAXS at $\alpha_{i}>\alpha_{c}$, because of the non constant scattered $q_{\perp}$ portion to $\hat{C}_{w}^{\text {Sphere }}$ (Eq. (7)). Therefore we do not recommend to use such kind of BA based PDI approaches to estimate particle polydispersity in GISAXS for $\alpha_{i}>\alpha_{c}$.

In case of weakly correlated structures the unified intensity can be correlated with a common structure factor $S(q)$

$$
I_{\text {corr }}(q)=I(q) S(q)
$$

$S(q)$ is a function, which describes the correlation between two structures with a centre to centre distance $\xi$ and a packing factor $\kappa[15,18]$ :

$$
S(q)=\frac{1}{1+\kappa F(q, \xi)} .
$$

The packing factor $\kappa$ describes the degree of correlation. For a perfect three dimensional crystal one yields $\kappa_{\max }=$ 5.92 , while for a perfect two dimensional crystal $\kappa_{\max }=$ 6.24 is obtained. $F(q, \xi)$ is the "form factor" for structural correlations occurring at an average distance $\xi[15]$

$$
F(q, \xi)=3 \frac{\sin (q \xi)-q \xi \cos (q \xi)}{(q \xi)^{3}}
$$

Equations (12)-(14) can be used only in the case of weakly correlated systems, when $I\left(q, R_{g}\right)$ can be decoupled from $S(q)$ and $S(q)$ is not dependent on $R_{g}$. This is usually the case for low correlations at $\kappa<4$.
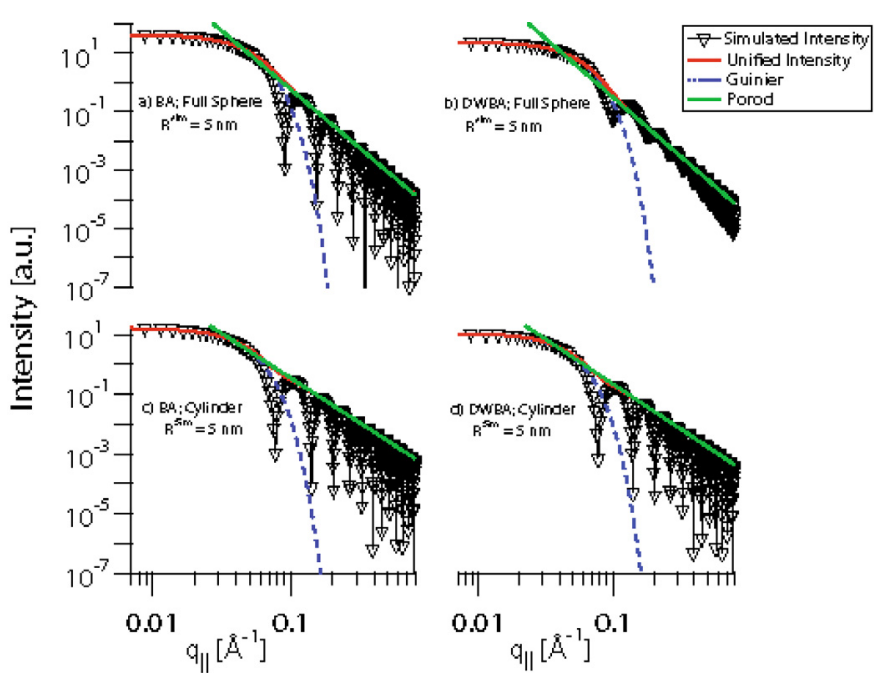

Fig. 2. (Color online) Comparison of $q_{\|}$scans from IsGISAXS simulated intensity using BA and DWBA with calculated unified fit intensity using different particle island geometries. (a) Monodisperse full sphere $\left(R^{\mathrm{Sim}}=5 \mathrm{~nm} ; R_{q}^{\mathrm{Sim}}=3.87 \mathrm{~nm}\right.$. (b) Monodisperse cylinder $\left(R_{\|}^{\mathrm{Sim}}=5 \mathrm{~nm} ; R_{c}^{\mathrm{Sim}}=3.53 \mathrm{~nm}\right)$.

\subsection{Comparison with simulations ${ }^{2}$}

In this chapter the adaptability of the unified fit approach to $q_{\|}$dependent GISAXS is tested. Simulated $q_{\|}$scans were fitted with the unified fit approach and deviations from DWBA to BA theory are discussed. To test the pictured BA approach for common particle types GISAXS from spherical and cylindrical particle shapes were simulated following Gaussian distributions with $\sigma=0-0.6$. IsGISAXS simulation results using BA and DWBA are compared with calculated intensity from the unified fit model using the free accessible Irena software ${ }^{3}$ [37] for Igor Pro, Wavemetrics Inc.

As a first step simulations, which mimic diffuse surface scattering from monodisperse, smooth particle islands supported on homogenous surfaces were performed. Such a model is used in the experimental section to describe the lateral correlations of rough Au films. Isotropic spherical particles were simulated with $R^{\mathrm{Sim}}=R_{x}=$ $R_{y}=R_{z}=5 \mathrm{~nm}$ (Figs. 2a, 2b). Particle islands of cylindrical symmetries were simulated with $R_{\|}^{\mathrm{Sim}}=R_{x}=$ $R_{y}=5$ (Figs. 2c, 2d). $R_{g}^{\mathrm{Sim}}$ and $R_{c}^{\mathrm{Sim}}$ were calculated according to $R_{g}^{\mathrm{Sim}}$ (Sphere) $=\sqrt{3 / 5} R^{\mathrm{Sim}}$ (Sphere) and $R_{c}^{\mathrm{Sim}}$ (Cylinder) $=\frac{1}{\sqrt{2}} R_{\|}^{\mathrm{Sim}}$ (Cylinder). Details of the parameters used in IsGISAXS simulations are given in the

\footnotetext{
${ }^{2}$ Supporting information: IsGISAXS simulations presented in this section are based on input data files, which contain all the specific parameters. Supporting information 1 contains a set of parameters on which GISAXS from monodisperse cylindrical particle islands was simulated. Supporting information 2 contains a set of parameters for GISAXS of monodisperse spherical particles buried in a typical polymer matrix.

3 www.usaxs.xor.aps.anl.gov/staff/ilavsky/irena. html
} 
Table 1. Unified fit results for particle types simulated in Figure 2 using BA and DWBA.

\begin{tabular}{lcccc}
\hline & \multicolumn{2}{c}{ Full sphere } & \multicolumn{2}{c}{ Cylinder } \\
\hline & BA & DWBA & BA & DWBA \\
$G$ & 42.7 & 23.4 & 17.0 & 11.3 \\
$R_{g}(\AA)$ & 41.5 & 38.0 & & \\
$R_{c}(\AA)$ & & & 37.7 & 37.8 \\
$B$ & $5.6 \times 10^{-5}$ & $3.0 \times 10^{-5}$ & $3.5 \times 10^{-4}$ & $2.2 \times 10^{-4}$ \\
$P$ & 4 & 4 & 3 & 3 \\
\hline
\end{tabular}

supplementary information. For BA simulated $q_{\|}$scans (similar to transmission SAXS geometry) incident and exit angles $\alpha_{i}$ and $\alpha_{f}$ were set to $0^{\circ}$, respectively. For DWBA simulated GISAXS the incident angle was set to $\alpha_{i}=0.7^{\circ}$, equal to the performed GISAXS experiments on the studied $\mathrm{Au}$ film, while the exit angle was set to $\alpha_{f}=\alpha_{c}(\mathrm{Au})=0.50^{\circ}$. Simulated $q_{\|}$scans of spherical particle islands (Figs. 2a, 2b) were compared with three dimensional averaged intensities from equation (8), while simulated $q_{\|}$scans of cylindrical particles (Figs. 2c, 2d) were compared with radial averaged intensities from equation (9).

The observed power-law decays of $q^{-4}$ for spherical particle islands and of $q^{-3}$ for radial averaged cylindrical particle islands are addressed to Porod surface scattering as described in the theoretical discussion. Oscillations, resulting from the monodisperse form factor and (in the DWBA) a coherent interference of reflected, refracted and scattered waves, vary from BA to DWBA. However, results of $R_{g}^{\mathrm{Fit}}$ and $R_{c}^{\mathrm{Fit}}$ from Guinier fits (Tab. 1) follow the employed parameters $R_{g}^{\mathrm{Sim}}$ and $R_{c}^{\mathrm{Sim}}$ with deviations $<10 \%$ for BA simulated transmission SAXS and DWBA simulated GISAXS. In contrast to $R_{g}$ and $R_{c}$ the obtained ratios of $B / G$ appear to be highly dependent on the scattering geometry and the applied perturbation theory (e.g. BA and DWBA). Consequently in GISAXS analysis at $\alpha_{i}>\alpha_{c}$ one should avoid BA based PDI approaches, which are commonly used in SAXS analysis.

Especially for spherical particles one may await deviations of $R_{g}$, when DWBA theory is approximated with BA. As expected, the discrepancies of the unified fit results from simulated values are dependent on $\alpha_{i}, R$ and $\sigma$ (Fig. 3). For monodisperse particle islands the quality of the applied fit is in the range of $10 \%$ over a large area in the contour diagram. However, for $\alpha_{i} \geq 1.0$ the fit quality reduces especially for $R \geq 7 \mathrm{~nm}$. This observation can be best explained by dominating refraction effects at high $\alpha_{i}$. For the simulated conditions the penetration depth into the film material was calculated to be $11 \mathrm{~nm}[38,39]$. For small particle sizes of $R<7 \mathrm{~nm}$ the beam's pathway in the particle is small and deviations occurring refraction are small. For $R \geq 7 \mathrm{~nm}$ the beam's pathway in the $\mathrm{Au}$ particles becomes longer, corresponding refraction effects are enhanced and deviations from BA, disregarding beam refraction, become larger.

Contour diagrams for polydisperse particle islands show enhanced fit discrepancies compared to monodisperse scatterers. Positive deviations towards higher $R_{g}^{\text {Fit }}$
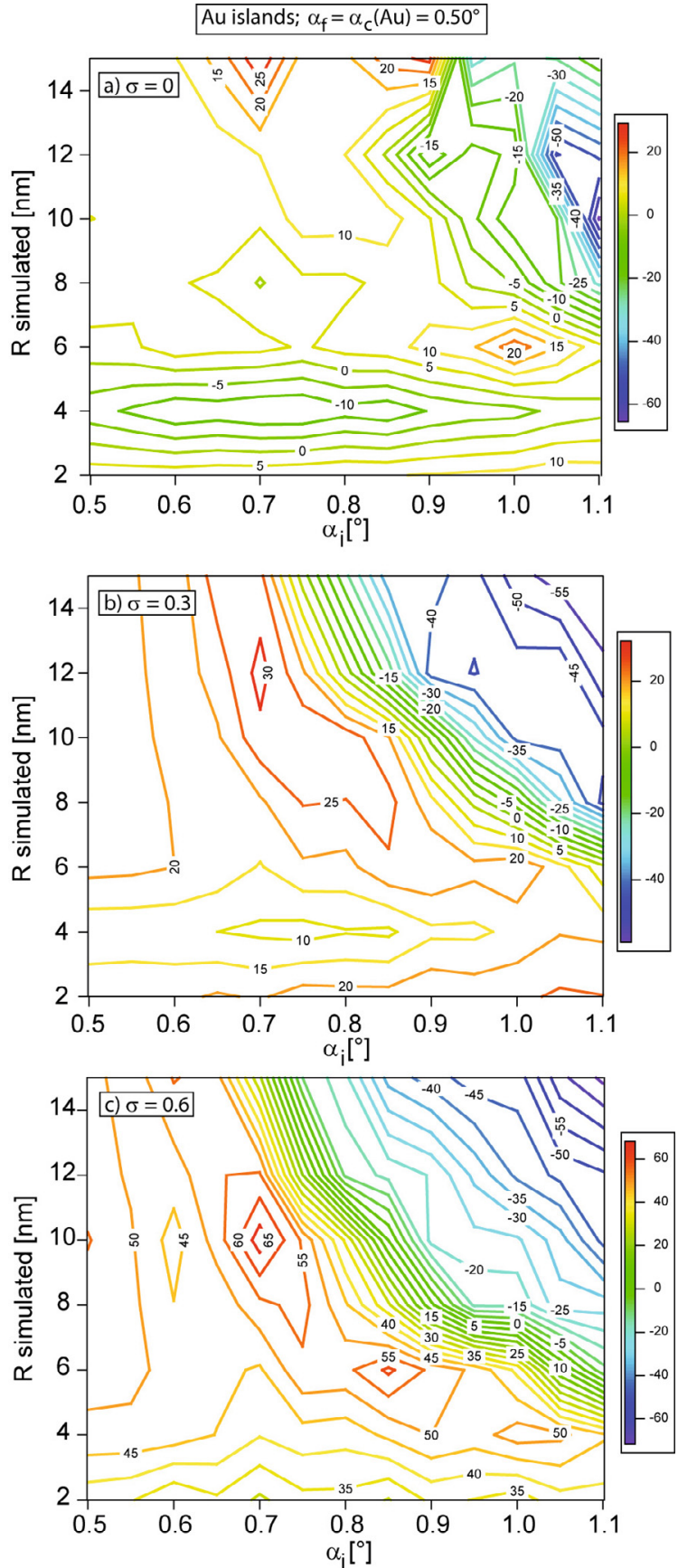

Fig. 3. (Color online) Contour plots of unified fit results from DWBA simulated $q_{\|}$scans at $\alpha_{f}=\alpha_{c}(\mathrm{Au})$ (Yoneda maximum) for $\mathrm{Au}$ particle islands in dependence of $R_{g}$ and $\alpha_{i}$ for different polydispersities. Contour lines represent the deviation of $R_{g}^{\text {Fit }}$ from $R_{g}^{\mathrm{Sim}}$ in percentage.

values indicate higher refraction contributions of big particles to GISAXS. Especially for $\sigma=0.6$ (Fig. 3c) the observed deviations seem to be high. However, in highly polydisperse particle samples defined spherical symmetries are usually difficult to achieve and knowledge of particle dimensions with mean deviations in the order of the system's polydispersity is in many cases sufficient. Concluding simulation results from Figure 3, acceptable 


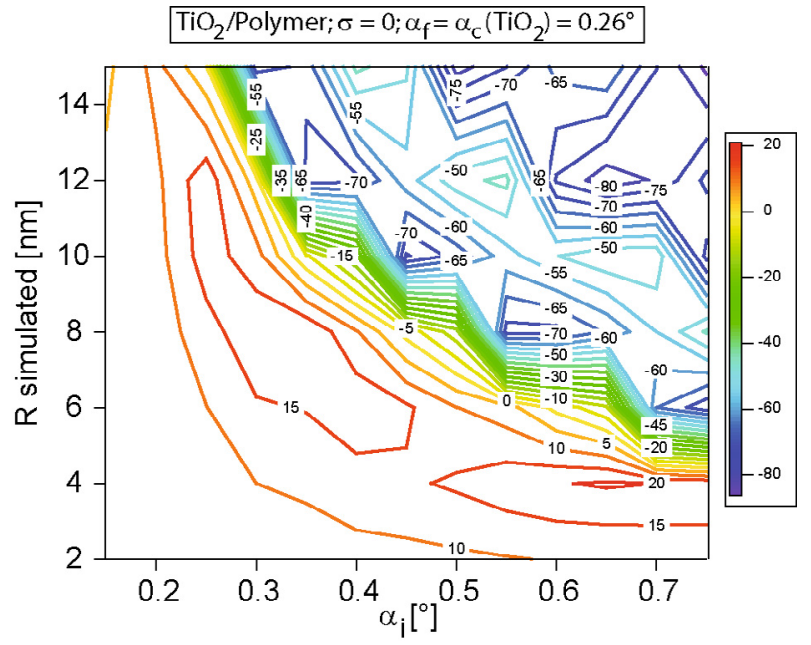

Fig. 4. (Color online) Contour plot of unified fit results from DWBA simulated $q_{\|}$scans at $\alpha_{f}=\alpha_{c}\left(\mathrm{TiO}_{2}\right)$ in dependence of $R_{g}$ and $\alpha_{i}$. Contour lines represent the deviation of $R_{g}^{\mathrm{Fit}}$ from $R_{g}^{\mathrm{Sim}}$ in percentage.

unified fit results for particle island systems are obtained for GISAXS experiments, conducted in the range of $0.7^{\circ} \leq$ $\alpha_{i} \leq 0.85^{\circ}$, corresponding to $1.4 \alpha_{c}(\mathrm{Au}) \leq \alpha_{i} \leq 1.7 \alpha_{c}$ $(\mathrm{Au})$. Within the GISAXS sample to detector distances used in our experimental set-up, incident angles of $\alpha_{i} \ll$ $0.7^{\circ}$ are usually not recommended, due to overlap of specular scattering and off specular scattering in the Yoneda peak region. However for higher sample to detector distances as used in GIUSAXS, $\alpha_{i} \ll 0.7^{\circ}$ could be used for better fit results.

Similar to simulations from particle islands, diffuse GISAXS of $\mathrm{TiO}_{2}$ particles buried in a polymer film matrix was simulated in dependence of $\alpha_{i}, R$ and $\sigma$. For the simulated two component film system, $q_{\|}$scans were performed at $\alpha_{f}=\alpha_{c}\left(\mathrm{TiO}_{2}\right)=0.26^{\circ}$ and at the Yoneda's maximum $\left(\alpha_{f}=0.15^{\circ}\right)$, which is near $\alpha_{c}$ of the matrix polymer $\left(\alpha_{c}=0.15^{\circ}\right)$. Transverse $q_{\|}$scans at $\alpha_{f}=$ $0.26^{\circ}$, show fit discrepancies up to $80 \%$ from the expected value. Fitting errors were only found to be acceptable for $R<4 \mathrm{~nm}$ or $\alpha_{i}<0.26^{\circ}$. Using arguments from particle island systems, refraction effects, which are not included in BA, become more important for high $\alpha_{i}$ and increasing $R$. Within the simulated conditions the polymer matrix of $50 \mathrm{~nm}$ is totally transparent and the possible penetration depth into the particle phase is $30 \mathrm{~nm}$. Thus refracted and reflected waves at all film interfaces interfere with particle scattered waves. These effects are less pronounced for small particles or small $\alpha_{i}$, where the beam's pathway in the particle phase is small.

Smaller deviations from DWBA simulations were obtained for $q_{\|}$scans at the Yoneda's maximum at $\alpha_{f}=$ $0.15^{\circ}$ (Fig. 5). At these conditions the polymer matrix is still transparent for the incoming beam, while the penetration depth into the particle phase drops to $6 \mathrm{~nm}$. For particles bigger than the penetration depth, the beam is not transmitted to the polymer/substrate interface. Consequently the superposition of polymer/substrate reflected
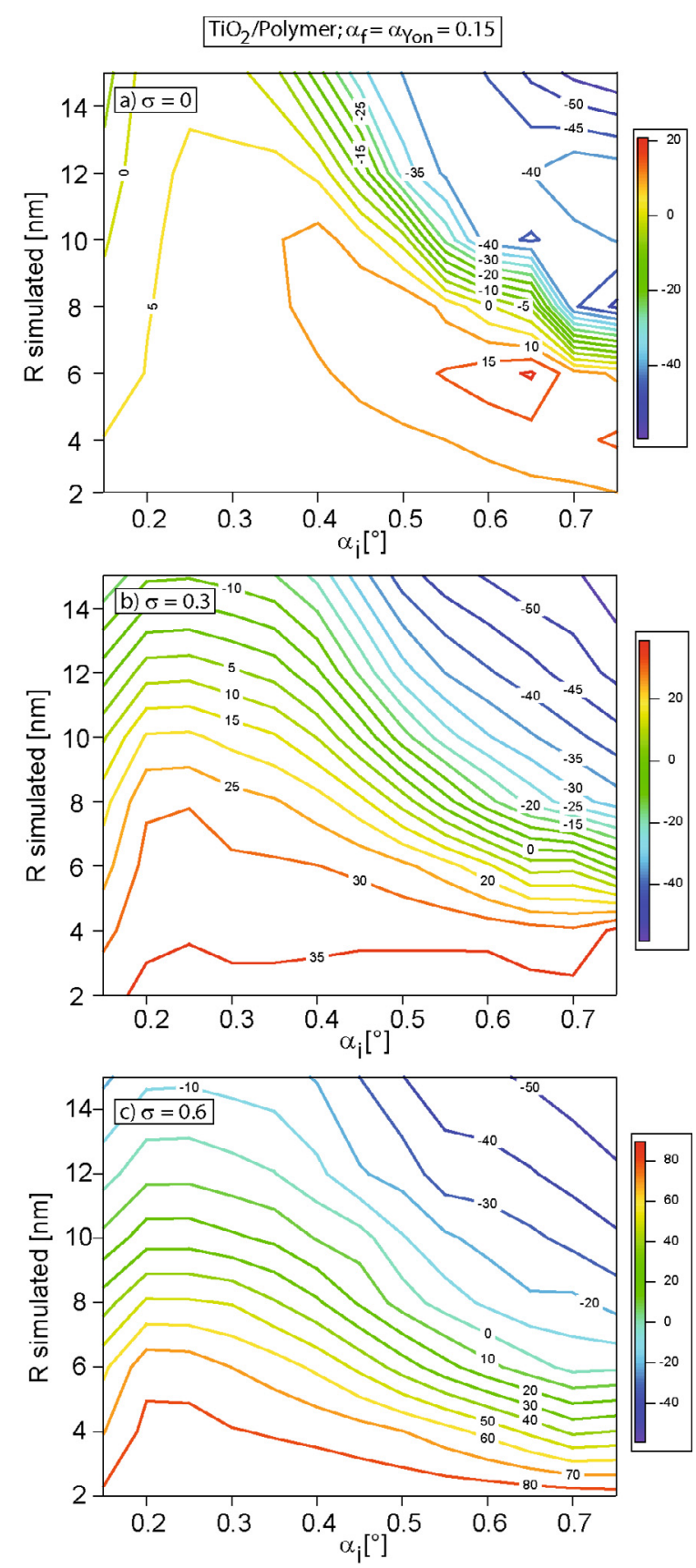

Fig. 5. (Color online) Contour plots of unified fit results from DWBA simulated $q_{\|}$scans at $\alpha_{f}$ equal the Yoneda maximum (near $\alpha_{c}$ (Polymer)) for $\mathrm{TiO}_{2}$ particle systems buried in a polymer matrix in dependence of $R_{g}$ and $\alpha_{i}$ for different polydispersities. Contour lines represent the deviation of $R_{g}^{\mathrm{Fit}}$ from $R_{g}^{\mathrm{Sim}}$ in percentage.

wave and scattered wave is minimized. Similar to Au particle island systems deviations become larger due to enhanced refraction for increasing particle sizes and incident angles.

Polydisperse systems show deviations towards higher $R_{g}^{\text {Fit }}$ values comparable to particle island systems. In contrast, fit deviations for buried particle systems are most 


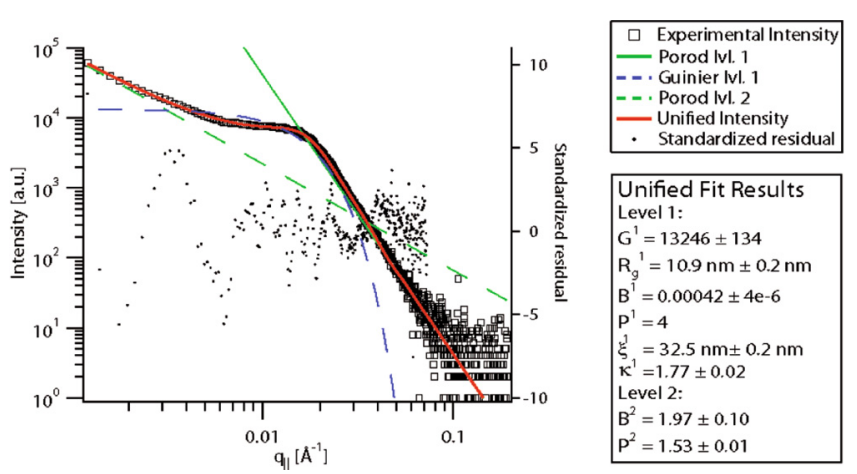

Fig. 6. (Color online) Experimental GISAXS data of low correlated Au film prepared by chemical vapour deposition fitted with equations (10), (12)-(14).

distinctive for small particles. However, deviations in the range of $2 \mathrm{~nm}$ for such particle dimensions are not crucial in many experimental cases, since the usual low signal to noise ratio at high $2 \theta$ angles do not allow fits more precise than $1 \mathrm{~nm}$.

Defining optimum experimental conditions we suggest incident angles in the range of $0.35^{\circ} \leq \alpha_{i} \leq 0.55^{\circ}$ corresponding to $2.5 \alpha_{c}$ (Polymer) $\leq \alpha_{i} \leq \overline{3} .9 \alpha_{c}$ (Polymer).

\subsection{Experimental discussion}

\subsubsection{Unified analysis from rough Au films}

Figure 6 shows experimental $q_{\|}$GISAXS data at $\alpha_{f} \approx$ $\alpha_{c}(\mathrm{Au})=0.5^{\circ}$ obtained from the $\mathrm{Au}$ film measured at $\alpha_{i}=0.7^{\circ}$, which is in the proposed angular range. Studying experimental data transverse scattering from two structural levels, which are separated by a correlation peak, can be observed. The found Porod decay of $\sim 4$ at high $q$ suggests the presence of particle islands, which can be approximated with the form of a hemisphere. Therefore, the use of the three dimensional averaged equation (10) can be used. From fit results of the 1st structural level, one can assign the average lateral dimension of the $\mathrm{Au}$ particles of $R_{g}^{1}=10.9 \mathrm{~nm}$. Results from the correlation functions (Eqs. (12)-(14)) included in the applied fit suggest that the covering particle islands are randomly arranged - as deduced from the low packing factor $\kappa^{1}=1.8$ - with a mean centre to centre distance of $\xi^{1}=33 \mathrm{~nm}$. In our experimental $q_{\|}$range we were only able to record few data-points, which account for Porod scattering of level 2. However, the found power law decay of $P^{2} \approx 1.5$ can give evidence to a mass fractal, which is related to a two dimensional arrangement of $\mathrm{Au}$ particle islands. GISAXS $q_{\|}$scans from the studied $\mathrm{Au}$ film and corresponding unified fit results were compared with comparative SPM data from randomly distributed $\mathrm{Au}$ islands (Fig. 7). Modelling them as hemispherical islands, mean particle island sizes of $R=13 \mathrm{~nm}$ corresponding to $R_{g}=10 \mathrm{~nm}$ with a $\sigma=0.27$ can be assigned. Accordingly a fit discrepancy towards SPM results of $10 \%$ can be assigned, which is even better than the error estimated by simulations. For

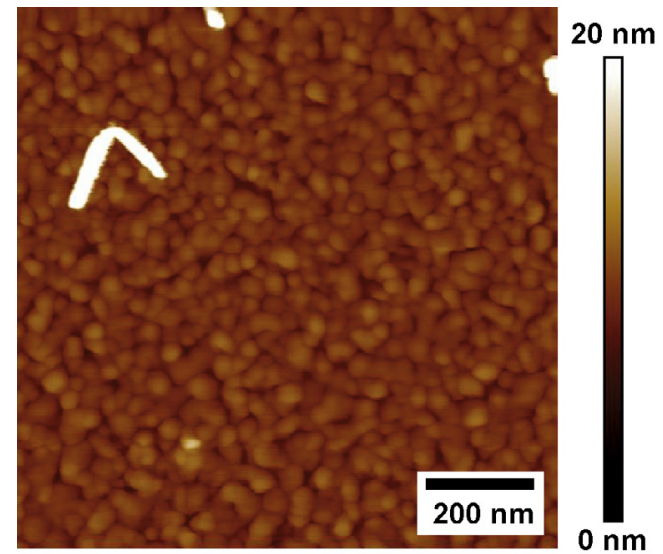

Fig. 7. (Color online) SPM image of the $20 \mathrm{~nm}$ thick Au film.

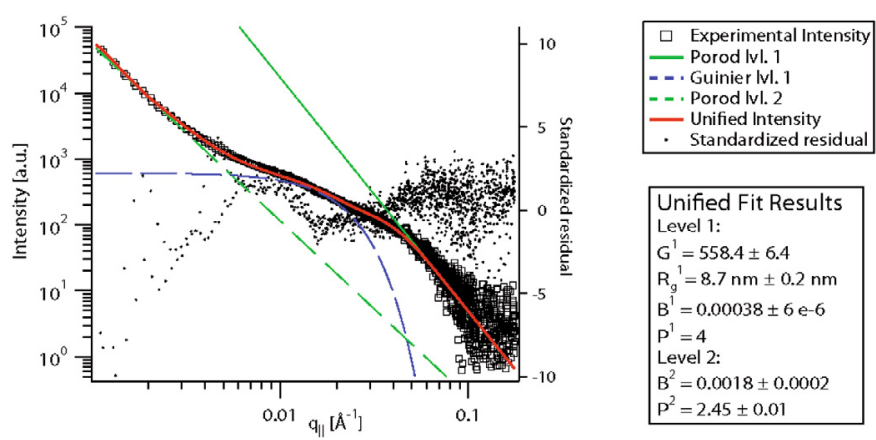

Fig. 8. (Color online) Experimental GISAXS data of uncorrelated spin coated $\mathrm{TiO}_{2}$ /PMMA film fitted with equation (10).

islands centre to centre correlation lengths mean values of $\xi=32 \mathrm{~nm} \pm 10 \mathrm{~nm}$ were obtained. The average particle island distance of $32 \mathrm{~nm}$ with the calculated standard deviation of $\sigma=0.32$ is in good agreement with the average distance and the low degree of correlation $\kappa$, found by GISAXS experiments.

\subsubsection{Unified analysis from $\mathrm{TiO}_{2} /$ PMMA films}

To test the formalism for GISAXS on buried particle systems experimentally, $q_{\|}$scans deduced from GISAXS in combination with comparative SEM results from spincoated $\mathrm{TiO}_{2} /$ PMMA hybrid material films are studied. Figure 8 shows a $q_{\|}$detector scan at the Yoneda's maximum at $\alpha_{f}=0.15^{\circ}$, which is near $\alpha_{c}(\mathrm{PMMA})=0.14^{\circ}$ obtained from a GISAXS pattern taken at $\alpha_{i}=3.9 \alpha_{c}=$ $0.55^{\circ}$. At high $q_{\|}$a power law decay with $P^{1} \approx 4$ for diffuse Porod scattering of the first structural level, meaning the immersed $\mathrm{TiO}_{2}$ particles, with $R_{g}^{1}=8.7 \mathrm{~nm}$ can be found. Immersed $\mathrm{TiO}_{2}$ particles seem to be uncorrelated and far separated in the PMMA matrix. This led to negligibly small contributions of the interference function $S\left(q_{\|}\right)$ to the unified intensity and was therefore approximated as unity. From the 2nd level Porod approach a power law decay in the range of $2<P^{2}<3$ was found. This finding 


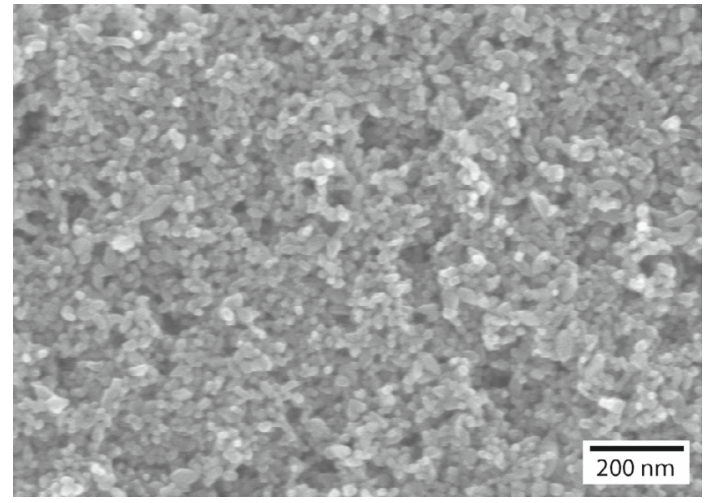

Fig. 9. SEM picture of the $\mathrm{TiO}_{2}$ particles (Solaronix T).

proposes a particle arrangement in an arbitrary three dimensional mass fractal. From comparison with SEM analysis (Fig. 9) a mean particle size of $R=9.2 \mathrm{~nm}$, corresponding to $R_{g}=7.1 \mathrm{~nm}$ with $\sigma=0.17$ was assigned. The corresponding deviation between SEM and BA approximated GISAXS is $23 \%$, which is in the order of the error range as proposed from simulations. To summarize GISAXS, SPM and SEM results: when transverse $q_{\|}$detector scans at $\alpha_{f}=\alpha_{c}$ are performed, predictions on particle island sizes, forms and arrangements including mass fractal dimensions can be made with the unified fit approach within a reasonable accuracy.

\subsubsection{Unified analysis from novel $\mathrm{TiO}_{2} /($ PEO)MA-PDMS-MA(PEO) films}

In this last experimental study the usefulness of the unified fit approach for clarifying morphologies in functional films containing polydisperse colloidal particles is demonstrated. Using conductive SPM it was suggested that electrical charges are transported through partly percolating $\mathrm{TiO}_{2} /(\mathrm{PEO}) \mathrm{MA}-\mathrm{PDMS}-\mathrm{MA}(\mathrm{PEO})$ networks. This allows for applications in solar cells [19]. A ceramized isolating PDMS shell prevents lateral shortcuts. For this material, high resolution SEM (Fig. 10) or SPM studies are unable to unravel the morphology of the film. Thus no clear conclusion on the charge transport mechanism can be made without additional experimental proof. In Figure 11 the analysed experimental GISAXS intensity of a $20 \mathrm{~nm}$ thick $\mathrm{TiO}_{2} /(\mathrm{PEO}) \mathrm{MA}-\mathrm{PDMS}-\mathrm{MA}(\mathrm{PEO})$ film taken at $\alpha_{i}=0.7^{\circ}$, is shown. The average $R_{g}$ of the primary uncorrelated $\mathrm{TiO}_{2}$ particles is found to be $4.0 \mathrm{~nm}$. Despite not being in the optimum angular range the dependence of the fit accuracy on $\alpha_{i}$ is not crucial for such small particles, as proposed by the presented simulations (Fig. 5). From $P^{2}=2.2$ can be concluded that the small particles are not two dimensionally arranged in the film matrix, but in a three dimensional mass fractal. Therefore the mechanism of charge transport can be specified to electrons percolating perpendicular to the film surface through a network of $\mathrm{TiO}_{2}$ particles. Particles are not correlated laterally, facilitating charge carrier transport perpendicular to the interface.

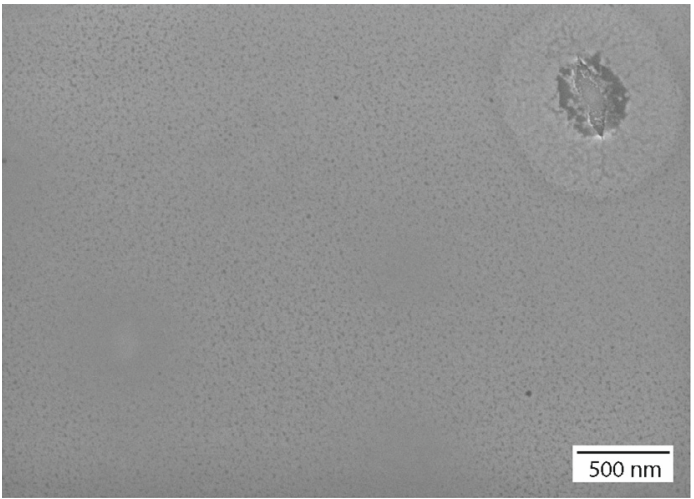

Fig. 10. SEM picture of the $\mathrm{TiO}_{2} /(\mathrm{PEO}) \mathrm{MA}-\mathrm{PDMS}-\mathrm{MA}$ (PEO) film.
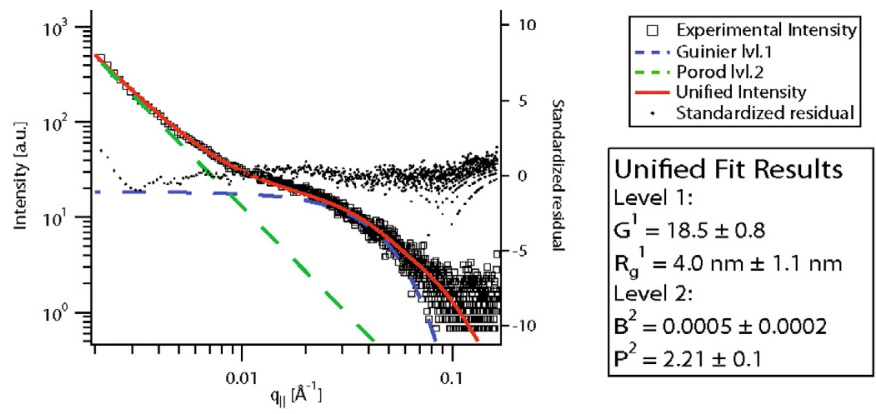

Fig. 11. (Color online) Experimental GISAXS data of spin coated $\mathrm{TiO}_{2} /(\mathrm{PEO}) \mathrm{MA}-\mathrm{PDMS}-\mathrm{MA}(\mathrm{PEO})$ film fitted with equation (10).

\section{Summary}

We have shown that the application of the unified fit equations, which were used over ten years for data analysis in transmission SAXS, USAXS and SLS, to transverse $q_{\|}$ detector scans in GISAXS experiments is allowed within certain limits. Theoretical consideration and simulations show that the unified formalism can be used for particles with spherical or cylindrical symmetry, either placed at the free surface or buried in a film matrix. Theoretical deviations in the range of the particles polydispersity are not critical for the analysis of typical colloidal particles. We concluded from simulations that experimental incident angles should be chosen carefully before conducting the GISAXS experiment. For our Au particle island film system best results are obtained for incident angles in the range of $1.4 \alpha_{c}(\mathrm{Au}) \leq \alpha_{i} \leq 1.7 \alpha_{c}(\mathrm{Au})$, while for $\mathrm{TiO}_{2}$ containing buried film systems best results are expected in the range of $2.5 \alpha_{c}$ (Polymer) $\leq \alpha_{i} \leq 3.9 \alpha_{c}$ (Polymer) with $\alpha_{f}$ at the Yoneda's maximum to guarantee acceptable results. However, this range may vary for different refractive indices. It was further shown that a BA approximation for small particles of $R \leq 6 \mathrm{~nm}$ is less dependent on experimental angles.

Comparing experimental GISAXS results with particle analysis from SPM and SEM studies, we were able to verify mean particle island sizes and centre to centre correlation lengths. In addition, further considerations 
on particle arrangements, described by packing factors and mass-fractal dimensions, were shown to be useful. A clear limit to the presented approach is that polydispersity approaches used in transmission SAXS [36], can be misleading for analysis of GISAXS at $\alpha_{i}>\alpha_{c}$ However, using the unified fit approach for GISAXS on novel functional films can help to unravel physical mechanisms where microscopic techniques like SEM or SPM would not be sufficient.

This research was supported by the Max Planck Society (Institutsübergreifende Forschungsinitiative FRM II), the DFG (GU771/2, MU1487/5) CSGI, MiUR and CNR-FUSINT. We gratefully thank the HASYLAB for provision of beamtime, M. Dommach, and R. Döhrmann for technical support at the beam line BW4, Dr. R. Berger for support with SPM and M. Müller for support with SEM.

\section{References}

1. P. Müller-Buschbaum, E. Bauer, E. Maurer, S.V. Roth, R. Gehrke, M. Burghammer, C. Riekel, J. Appl. Crystallogr. 40, S341 (2007)

2. P. Müller-Buschbaum, M. Casagrande, J. Gutmann, T. Kuhlmann, M. Stamm, G. von Krosigk, U. Lode, S. Cunis, R. Gehrke, Europhys. Lett. 42, 517 (1998)

3. R. Lazzari, G. Renaud, C. Revenant, J. Jupille, Y. Borensztein, Phys. Rev. B 79, 8 (2009)

4. Y. Yoneda, Phys. Rev. 131, 2010 (1963)

5. G.H. Vineyard, Phys. Rev. B 26, 4146 (1982)

6. T. Salditt, T.H. Metzger, J. Peisl, B. Reinker, M. Moske, K. Samwer, Europhys. Lett. 32, 331 (1995)

7. T. Salditt, T.H. Metzger, C. Brandt, U. Klemradt, J. Peisl, Phys. Rev. B 51, 5617 (1995)

8. S.K. Sinha, E.B. Sirota, S. Garoff, H.B. Stanley, Phys. Rev. B 38, 2297 (1988)

9. V. Holy, J. Kubena, I. Ohlidal, K. Lischka, W. Plotz, Phys. Rev. B 47, 15896 (1993)

10. V. Holy, T. Baumbach, Phys. Rev. B 49, 10668 (1994)

11. M. Rauscher, T. Salditt, H. Spohn, Phys. Rev. B 52, 16855 (1995)

12. M. Rauscher, R. Paniago, H. Metzger, Z. Kovats, J. Domke, J. Peisl, H.D. Pfannes, J. Schulze, I. Eisele, J. Appl. Phys. 86, 6763 (1999)

13. R. Lazzari, J. Appl. Crystallogr. 35, 406 (2002)

14. J.S. Pedersen, P. Vysckocil, B. Schonfeld, G. Kostorz, J. Appl. Crystallogr. 30, 975 (1997)
15. A. Guinier, G. Fournet, Small-Angle Scattering of X-rays (Wiley, New York, 1955)

16. G. Beaucage, J. Appl. Crystallogr. 28, 717 (1995)

17. G. Beaucage, J. Appl. Crystallogr. 29, 134 (1996)

18. G. Porod, Small-Angle X-ray Scattering (Academic Press, London, 1982)

19. M. Memesa, S. Weber, S. Lenz, J. Perlich, R. Berger, P. Muller-Buschbaum, J.S. Gutmann, Energy Environ. Sci. 2, 783 (2009)

20. R. Gehrke, Rev. Sci. Instrum. 63, 455 (1992)

21. S.V. Roth, M. Burghammer, C. Riekel, P. Muller-Buschbaum, A. Diethert, P. Panagiotou, H. Walter, Appl. Phys. Lett. 82, 1935 (2003)

22. S.V. Roth, R. Dohrmann, M. Dommach, M. Kuhlmann, I. Kroger, R. Gehrke, H. Walter, C. Schroer, B. Lengeler, P. Muller-Buschbaum, Rev. Sci. Instrum. 77, 085106 (2006)

23. S. Dietrich, A. Haase, Phys. Rep. 260, 1 (1995)

24. J.S. Gutmann, thesis, Johannes Gutenberg-Universität, 2000

25. A. Frömsdorf, R. Capek, S.V. Roth, J. Phys. Chem. B 110, 15166 (2006)

26. B.B. Mandelbrot, The Fractal Geometry of Nature (Freeman, New York, 1982)

27. J.P. Schlomka, M. Tolan, L. Schwalowsky, O.H. Seeck, J. Stettner, W. Press, Phys. Rev. B 51, 2311 (1995)

28. R. Chiarello, V. Panella, J. Krim, C. Thompson, Phys. Rev. Lett. 67, 3408 (1991)

29. G. Beaucage, S. Rane, S. Sukumaran, M.M. Satkowski, L.A. Schechtman, Y. Doi, Macromolecules 30, 4158 (1997)

30. S.V. Chavan, P.U. Sastry, A.K. Tyagi, Scripta Mater. 55, $569(2006)$

31. B.C. Tappan, M.H. Huynh, M.A. Hiskey, D.E. Chavez, E.P. Luther, J.T. Mang, S.F. Son, J. Am. Chem. Soc. 128, 6589 (2006)

32. S. di Stasio, J.B.A. Mitchell, J.L. LeGarrec, L. Biennier, M. Wulff, Carbon 44, 1267 (2006)

33. A.I. Norman, D.L. Ho, A. Karim, E.J. Amis, J. Colloid Interface Sci. 288, 155 (2005)

34. S. Rathgeber, T. Pakula, V. Urban, J. Chem. Phys. 121, 3840 (2004)

35. J.S. Higgins, H. Benoit, Polymers and Neutron Scattering (Oxford, 1994)

36. G. Beaucage, H.K. Kammler, S.E. Pratsinis, J. Appl. Crystallogr. 37, 523 (2004)

37. J. Ilavsky, P.R. Jemian, J. Appl. Crystallogr. 42, 347 (2009)

38. H. Dosch, B.W. Batterman, D.C. Wack, Phys. Rev. Lett. 56, 1144 (1986)

39. H. Dosch, Phys. Rev. B 35, 2137 (1987) 